\title{
DNA Profiling of Leuconostoc citreum Strains in Fermented Foods by Repetitive Element Polymerase Chain Reaction ${ }^{5}$
}

\author{
Jasmine Kaur, Anshul Sharma, Sulhee Lee, and Young-Seo Park* \\ Department of Food Science and Biotechnology, Gachon University, Seongnam 13120, Republic of Korea
}

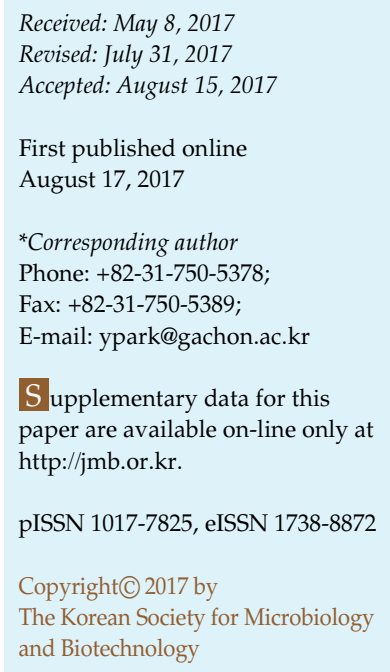

To identify and discriminate the bacterial species at the subspecific level, rep-PCR is a reliable genomic fingerprinting tool. Fourteen strains of bacteria were isolated from different food sources, identified as Leuconostoc citreum using 16S rRNA gene sequencing, and amplified using rep-primers (REP, ERIC, and $(\mathrm{GTG})_{5}$ ). Fingerprinting patterns generated bands in the range of 300-6,000 bp with REP, 150-6,000 bp with ERIC, and 200-1,700 bp with (GTG) primers. In UPGMA dendrogram analysis, 14 strains were clustered into three clades (I, II, and III) with all the primers, thus differentiating them at the molecular level. The present study revealed the differentiation of L. citreum strains using rep-PCR.

Keywords: Leuconostoc citreum, rep-PCR, ERIC-PCR, (GTG) $)_{5}$ PCR
The genus Leuconostoc is a Gram-positive, non-motile, catalase-negative, facultative anaerobe with a relatively low GC content (37-45 mol\%). It consists of 24 different species [1], an important one being Leuconostoc citreum, which has recently been established to have four genomic sequences [2]. Although genus Leuconostoc is found in human feces, vagina, and breast milk, it is not part of the human microbiota [3, 4]. However, it is extensively used in the production of cheese, butter, kimchi, sourdough, etc. [4, 5]. Along with other Leuconostoc spp., L. citreum provides the natural flavor and texture to kimchi (a Korean traditional dish) [6-8]. The classification and characterization of lactic acid bacteria (LAB) include phenotyping and genotyping. Compared with phenotypic methods, genotypic ones offer advantages such as general applicability and good discriminatory power [4,9]. Various methods have been used for typing of bacteria, amongst which repetitive element polymerase chain reaction (rep-PCR) is considered as one of the most robust techniques with high discriminatory power to identify species [10-13] and provide unambiguous results [14]. It amplifies the repetitive intergenic elements of the DNA, which provide information on the genetic diversity and population structure of the strain.

The objective of this study was to use rep-PCR for the characterization of South Korean L. citreum strains isolated from various food items. The 14 bacterial strains (Table 1) were cultured in MRS broth (Difco Laboratories, USA) at $37^{\circ} \mathrm{C}$ overnight. For DNA isolation and $16 \mathrm{~S}$ rRNA gene amplification, the protocol used has been described previously [5]. The 16S rRNA gene sequencing results identified the colonies as L. citreum (Accession Nos. KX286339-KX286352), and the UPGMA dendrogram covering 16S rRNA gene sequencing analysis for the $14 \mathrm{~L}$. citreum strains is shown in the supplementary material. The rep-PCR amplification was carried out as described previously [15] with REP-PCR (primer sets Rep1R-I and Rep2-I), ERIC-PCR (primer sets ERIC1R and ERIC2), and (GTG) $)_{5}$-PCR (primer (GTG) $)_{5}$ ) in a Bio-Rad thermal cycler (Bio-Rad Laboratories, USA) using $35 \mathrm{ng}$ of template DNA and $10 \mathrm{mM}$ concentration of primers (synthesized by Macrogen Inc., Korea). The amplified DNA fragments were separated by electrophoresis on a $1.5 \%$ agarose gel [16] with $100 \mathrm{bp}$ and $1 \mathrm{~kb}$ DNA ladders (TaKaRa Bio. Inc., Japan) as the size markers. The gel imaging was done with a gel documentation system (Gel Doc $\mathrm{XR}^{+}$; Bio- 
Rad Laboratories). Finally, the bands were scored 1 for the presence and 0 in the absence, and the dendrogram was

Table 1. Details of Leu. citreum strains from Korea.

\begin{tabular}{lll}
\hline Strain & Area/locality & \multicolumn{1}{c}{ Source } \\
\hline 11001 & Seongnam / Korea & Sweet pumpkin \\
11037 & Seongnam / Korea & Salted small octopus \\
11076 & Seongnam / Korea & Kimchi \\
11077 & Seongnam / Korea & Kimchi \\
11082 & Seongnam / Korea & Kimchi \\
11093 & Seongnam / Korea & Pear \\
11382 & Seongnam / Korea & Salted small octopus \\
11388 & Seongnam / Korea & Young reddish kimchi \\
11391 & Seongnam / Korea & Salted small octopus \\
11413 & Seongnam / Korea & Kimchi \\
11414 & Seongnam / Korea & Kimchi \\
11422 & Seongnam / Korea & Kimchi \\
11432 & Seongnam / Korea & Salted oyster \\
11447 & Seongnam / Korea & Salted fish \\
\hline
\end{tabular}

A

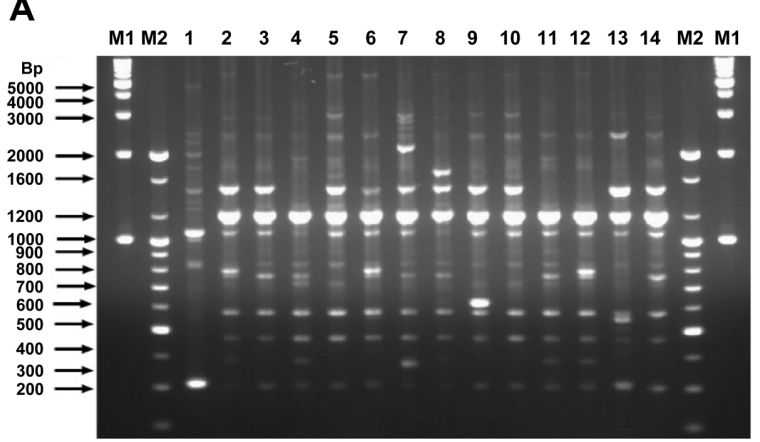

B

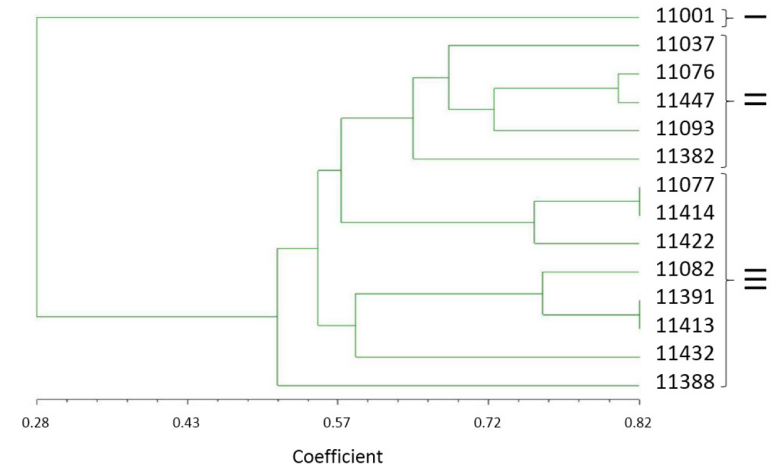

Fig. 1. (A) REP-PCR fingerprint profiles and (B) UPGMA dendrogram showing the different cluster divisions of 14 strains of Leuconostoc citreum isolated from food sources.

Lane M1: Marker 1 kb; Lane M2: Marker 100 bp; Lane 1: 11001; Lane 2: 11037; Lane 3: 11076; Lane 4: 11077; Lane 5: 11082; Lane 6: 11093; Lane 7: 11382; Lane 8: 11388; Lane 9: 11391; Lane 10: 11413; Lane 11: 11414; Lane 12: 11422; Lane 13: 11432; Lane 14: 11447. constructed with unweighted pair-group method with arithmetic mean method using Numerical Taxonomy System software [17].

The band patterns generated by the three primers were different and contrasting. In addition, phylogenetic trees verified the results obtained from the banding profiles of each strain. The strains were divided into three clades (I, II, and III) with all the primers. Most of the strains showed $\geq 40 \%,>60 \%$, and $>20 \%$ similarity with REP-PCR, (GTG) $)^{-}$ PCR, and ERIC-PCR, respectively.

For REP-PCR, 31 distinct fragments with 191 bands in total were observed with a band range of 300-6,000 bp (Fig. 1A). A maximum of 17 bands was found for strain 11082 (kimchi), and a minimum of 10 were found for strain 11001 (sweet pumpkin). However, REP-PCR showed the presence of 1,050 and $300 \mathrm{bp}$ bands for all 14 strains. A unique fingerprinting pattern was revealed for strain 11001, with the presence of bands at 100,1,400, 2,200, and 5,000 bp that were not seen in any other sample, along with the absence of bands at 490, 590, and 1,200 bp (Fig. 1A). There

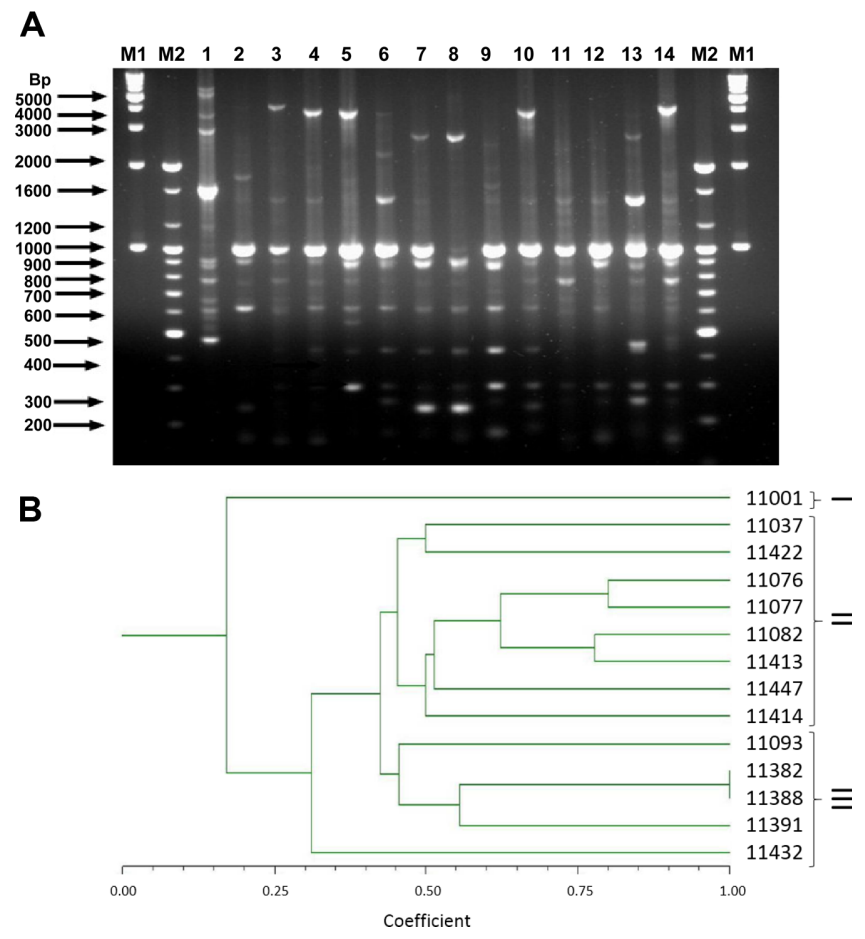

Fig. 2. (A) ERIC-PCR fingerprint profiles and (B) UPGMA dendrogram showing the different cluster divisions of 14 strains of Leuconostoc citreum isolated from food sources. Lane M1: Marker 1 kb; Lane M2: Marker 100 bp; Lane 1: 11001; Lane 2: 11037; Lane 3: 11076; Lane 4: 11077; Lane 5: 11082; Lane 6: 11093; Lane 7: 11382; Lane 8: 11388; Lane 9: 11391; Lane 10: 11413; Lane 11: 11414; Lane 12: 11422; Lane 13: 11432; Lane 14: 11447. 
were other uncommon bands observed in various strains that were not shared with the rest of them. Apart from this, the 1,100 bp band was found absent in strains 11001 and 11388. Interestingly, strain 11001 was seen to be distinguished in the other two cases as well. Phylogenetically, out of the three, clade III of REP-PCR contained strains 11077 and 11414 (Fig. 1B), which were closely related and isolated from a similar food source (i.e., kimchi), whereas strains 11391 and 11413 were found to be similar but were isolated from salted small octopus and kimchi, respectively.

However, ERIC primers generated 25 fragments with 110 bands in a range of $150-6,000 \mathrm{bp}$ (Fig. 2A). In contrast to the REP primer results, a maximum of 13 bands were obtained from strain 11001 with bands of 1,200, 2,900, 5,000 , and 6,000 bp, which were absent in the remaining strains. Moreover, the band at 1,000 bp was reported for all strains except for 11001. Phylogenetically, strain 11382 (salted small octopus) was categorized into clade III and showed a strong relationship with strain 11388 (young

\section{A}

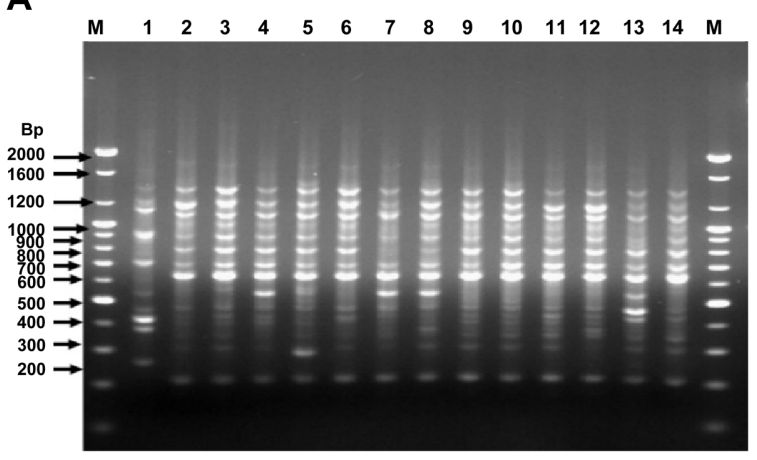

B

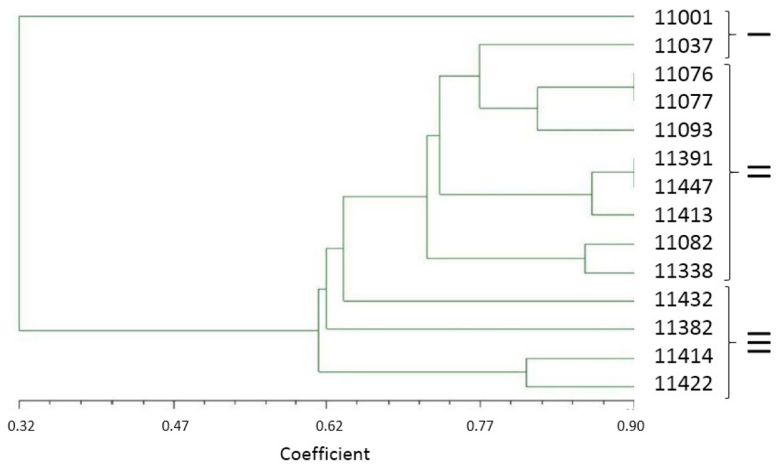

Fig. 3. (A) (GTG) $)_{5}$ PCR fingerprint profiles and (B) UPGMA dendrogram showing the different cluster divisions of 14 strains of Leuconostoc citreum isolated from food sources.

Lane M: Marker $1 \mathrm{~kb}$; Lane 1: 11001; Lane 2: 11037; Lane 3: 11076; Lane 4: 11077; Lane 5: 11082; Lane 6: 11093; Lane 7: 11382; Lane 8: 11388; Lane 9: 11391; Lane 10: 11413; Lane 11: 11414; Lane 12: 11422; Lane 13: 11432; Lane 14: 11447. radish kimchi) (Fig. 2B). It can be stated that strain 11382 had genetic relatedness with the strains isolated from kimchi.

The (GTG) $)_{5}$ primers showed 20 distinct fragments with a total of 167 bands in a range of 200-1,700 bp (Fig. 3A). Nevertheless, the highest number of bands (15) was found in strains 11082 (kimchi) and 11388 (young radish kimchi), and the minimum ( 9 bands) was seen in 11422 (kimchi). Bands of 450, 700, and 1,300 bp were observed for all the strains. Furthermore, bands of 200,600, and 1,100 bp were reported in all strains except 11001, whereas a $250 \mathrm{bp}$ band was only observed in strain 11001.

Unlike REP-PCR and ERIC-PCR, the phylogenetic analysis with (GTG) $)_{5}$-PCR revealed that from clade II, strains 11076 and 11077 (both sourced from kimchi) were identical, which were accompanied by strains 11391 and 11447 (isolated from salted small octopus and salted fish, respectively) that exhibited similarity (Fig. 3B). Interestingly, strain 11001 (sweet pumpkin) was represented in a single clade in all three PCR cases, suggesting that it carried a unique and distinctive profile from that of the other strains under study. However, unlike REP-PCR and (GTG) $)_{5}$ PCR, there was no band common to all strains in ERIC-PCR, but unique bands of 4,500 and $550 \mathrm{bp}$ were observed in strains, 11076 and 11082, respectively.

The different profiles generated can be attributed to the dispersed repetitive sequences in the canonical sequence $[12,18,19]$. Less similarity (more than $50 \%$ ) was observed among the strains from the same source (i.e., kimchi) and only a few were observed to be similar, which is supported by a study based on Leuconostoc strains from sourdough with distinct rep-PCR profiles [20]. Moreover, the differences may arise owing to the effect of the environment (home or industry) provided for kimchi fermentation. In this study, REP-PCR produced complex fingerprint banding patterns, followed by ERIC and then (GTG) $)_{5}$. This is in contrast to previous studies where the $(\mathrm{GTG})_{5}$ primer was found to be the most suitable for identifying Leuconostoc species [20, 21] and other LAB [10, 16, 22, 23]. However, several studies have revealed distinctive results with different primers. Comparable profiles were seen with REP, BOX, and (GTG) primers in a study based on the identification of probiotic strains [23], whereas ERIC-PCR was found to generate more complex patterns than REP-PCR in differentiating Lactobacillus johnsonii strains [9]. Even the less complex patterns make it easier to analyze the results [24,25], which helped toward understanding the genetic relatedness among the strains [16, 26, 27]. Overall, the ability of rep-PCR to show variations at the strain level proved it to be a reliable technique in the characterization of Gram-positive LAB. 
Rep-PCR is an easy, rapid, reliable, and reproducible molecular typing method with a practical approach. To our best knowledge, there is no other study that has differentiated L. citreum strains only by comparative analysis with repPCR.

\section{Acknowledgments}

This work was supported by the Korea Institute of Planning and Evaluation for Technology in Food, Agriculture, Forestry and Fisheries (IPET) through the High Value-added Food Technology Development Program, funded by the Ministry of Agriculture, Food and Rural Affairs (MAFRA; Grant No. 314073-03-2-HD040).

\section{References}

1. Kot W, Neve H, Heller KJ, Vogensen FK. 2014. Bacteriophages of Leuconostoc, Oenococcus, and Weissella. Front. Microbiol. 5: 186.

2. Passerini D, Vuillemin M, Laguerre S, Amari M, Loux V, Gabriel V, et al. 2014. Complete genome sequence of Leuconostoc citreum strain NRRL B-742. Genome Announc. 2: e1179-14.

3. Hemme D, Foucaud-Scheunemann C. 2004. Leuconostoc, characteristics, use in dairy technology and prospects in functional foods. Int. Dairy J. 14: 467-494.

4. Dan T, Liu W, Sun Z, Lv Q, Xu H, Song Y, et al. 2014. A novel multi-locus sequence typing (MLST) protocol for Leuconostoc lactis isolates from traditional dairy products in China and Mongolia. BMC Microbiol. 14: 150-158.

5. Kaur J, Lee S, Park YS, Sharma A. 2017. RAPD analysis of Leuconostoc mesenteroides strains associated with vegetables and food products from Korea. LWT Food Sci. Technol. 77: 383-388.

6. Choi IK, Jung SH, Kim BJ, Park SY, Kim J, Han HU. 2003. Novel Leuconostoc citreum starter culture system for the fermentation of kimchi, a fermented cabbage product. Antonie Van Leeuwenhoek 84: 247-253.

7. Lee JS, Heo GY, Lee JW, Oh YJ, Park JA, Park YH, et al. 2005. Analysis of kimchi microflora using denaturing gradient gel electrophoresis. Int. J. Food Microbiol. 102: 143-150.

8. Chang JY, Chang HC. 2010. Improvements in the quality and shelf life of kimchi by fermentation with the induced bacteriocin-producing strain, Leuconostoc citreum GJ7 as a starter. J. Food Sci. 75: 103-110.

9. Ventura M, Zink R. 2002. Specific identification and molecular typing analysis of Lactobacillus johnsonii by using PCR-based methods and pulsed-field gel electrophoresis. FEMS Microbiol. Lett. 217: 141-154.

10. Tamang JP, Tamang B, Schillinger U, Franz CMAP, Gores M, Holzapfel WH. 2005. Identification of predominant lactic acid bacteria isolated from traditionally fermented vegetable products of the Eastern Himalayas. Int. J. Food Microbiol.
105: 347-356.

11. Kingston JJ, Radhika M, Roshini MA, Murali HS, Batra HV. 2010. Molecular characterization of lactic acid bacteria recovered from natural fermentation of beet root and carrot Kanji. Indian J. Microbiol. 50: 292-298.

12. Muiru WM, Koopmann B, Tiedemann AV, Mutitu EW, Kimenju JW. 2010. Use of repetitive extragenic palindromic (REP), enterobacterial repetitive intergenic consensus (ERIC) and BOX sequences to fingerprint Exserohilum turcicum isolates. J. Appl. Biosci. 30: 1828-1838

13. Tafvizi F, Ebrahimi MT. 2015. Application of repetitive extragenic palindromic elements based on PCR in detection of genetic relationship of lactic acid bacteria species isolated from traditional fermented food products. J. Agric. Sci. Technol. 17: 87-98.

14. Olive DM, Bean P. 1999. Principles and applications of methods for DNA based typing of microbial organisms. J. Clin. Microbiol. 37: 1661-1669.

15. Versalovic J, Schneider M, Bruijn FJ, Lupski JR. 1994. Genomic fingerprinting of bacteria using repetitive sequence based polymerase chain reaction. Methods Mol. Biol. 5: 25-40.

16. Gevers D, Huys G, Swings J. 2001. Applicability of rep-PCR fingerprinting for identification of Lactobacillus species. FEMS Microbiol. Lett. 205: 31-36.

17. Faridi F, Sedigheh J. 2015. REP-PCR typing, antibiogram pattern and distribution of clinical isolates of Pseudomonas aeruginosa in a teaching hospital in South of Iran. Mol. Med. J. 1: 47-55.

18. Rademaker JLW, Hoste B, Louws FJ, Kersters K, Swings J, Vauterin L, et al. 2000. Comparison of AFLP and rep-PCR genomic fingerprinting with DNA-DNA homology studies: Xanthomonas as a model system. Int. J. Syst. Evol. Microbiol. 50: 665-677.

19. Lee CM, Sieo CC, Cheah YK, Abdullah N, Ho YW. 2012. Discrimination of probiotic Lactobacillus strains for poultry by repetitive sequenced based PCR fingerprinting. J. Sci. Food Agric. 92: 660-666.

20. Bounaix M-S, Gabriel V, Robert H, Morel S, RemaudSimeon M, Gabriel B, et al. 2010. Characterization of glucanproducing Leuconostoc strains isolated from sourdough. Int. J. Food Microbiol. 144: 1-9.

21. Tamang B, Tamang JP, Schillinger U, Franz CMAP, Gores M, Holzapfel WH. 2008. Phenotypic and genotypic identification of lactic acid bacteria isolated from ethnic fermented bamboo tender shoots of North East India. Int. J. Food Microbiol. 121: 35-40.

22. Stephenson DP, Moore RJ, Allison GE. 2009. Comparison and utilization of repetitive-element PCR techniques for typing Lactobacillus isolates from the chicken gastrointestinal tract. Appl. Environ. Microbiol. 75: 6764-6776.

23. Coudeyras S, Marchandin H, Fajon C, Forestier C. 2008. Taxonomic and strain-specific identification of the probiotic strain Lactobacillus rhamnosus 35 within the Lactobacillus casei 
group. Appl. Environ. Microbiol. 74: 2679-2689.

24. Rademaker JLW, de Bruijn FJ. 1997. Characterization and classification of microbes by rep-PCR genomic fingerprinting and computer assisted pattern analysis, pp. 151-171. In CaetanoAnollés G, Gresshoff PM (eds.), DNA Markers: Protocols, Applications and Overviews. Willey-Liss Inc., New York.

25. Baldy-Chudzik K, Niedbach J, Stosik M. 2003. Rep-PCR fingerprinting as a tool for the analysis of genomic diversity in Escherichia coli strains isolated from an aqueous/freshwater environment. Cell. Mol. Biol. Lett. 8: 793-798.
26. Vuyst LD, Camu N, Winter TD, Vandemeulebroecke K, Perre VV, Vancanneyt $\mathrm{M}$, et al. 2008. Validation of the (GTG)5-rep-PCR fingerprinting technique for rapid classification and identification of acetic acid bacteria, with a focus on isolates from Ghanaian fermented cocoa beans. Int. J. Food Microbiol. 125: 79-90.

27. Soltysik DA, Bednarek IA, Loch TM, Galka SE, Sypniewski DJ, Machnik GM, et al. 2010. Repetitive extragenic palindromic PCR (REP-PCR) as an alternative method for detection of bulking in activated sludge. Pol. J. Microbiol. 59: 11-20. 